babies died, one had normal levels of H.P.L., one had higher than normal levels, and one had low levels. These authors were therefore unimpressed with the general value of such determinations.

The chief contender among diagnostic tests for placental insufficiency is the determination of urinary oestriol levels. This is now well established, and in contrast to H.P.L. it measures the function of the foetus and placenta together as a unit. However, if measurement of H.P.L. proves to be of similar value, then it offers the advantages that it can be performed on single blood samples and that larger numbers of tests can be carried out. But the evidence on its value is conflicting and further work on it is needed.

\section{Massive Haemoptysis}

It is perhaps not widely recognized that when haemoptysis is massive death is common. ${ }^{1-4}$ The idea that reassurance by the physician is the best treatment, since even copious haemorrhages will cease in time, ${ }^{5}$ may encourage the view that conservative management is all that is required. But a study reported by J. A. Crocco and his colleagues ${ }^{1}$ is a timely reminder that massive haemoptysis is serious and potentially fatal. With massive haemoptysis defined as the loss of 600 $\mathrm{ml}$. or more of blood within $\mathbf{4 8}$ hours, overall mortality among 67 patients was $37 \%$; if blood-loss was at the rate of more than $600 \mathrm{ml}$. in 16 hours, it rose to $75 \%$. These figures emphasize the dangers in too much reliance on conservative management.

It is difficult to be precise about the relative frequency of the causes of massive haemoptysis, because reported series are often biased in one direction or another, but tuberculosis (both active and inactive) is certainly prominent, while lung abscess, bronchiectasis, and bronchial carcinoma are other common causes. Necrotizing arteritis has also been associated with massive haemoptysis, ${ }^{6} 7$ and bleeding from lung abscess seems to be particularly lethal. ${ }^{18}$ Rarer causes are broncholiths, pulmonary infarct, trauma, and mycetomata in association with pulmonary aspergillosis. Massive bleeding may also occur in patients with a tracheostomy tube in situ. Crocco and his colleagues showed that there was no significant variation in mortality according to age and also that $87 \%$ of their patients had had previous episodes of haemoptysis. Further analysis showed that the mortality increased with the rate of bleeding, so it is clearly important to avoid delay in making decisions about treatment.

Massive haemoptysis must be regarded as a medical emergency and calls for immediate investigation of the patient. Early chest radiography is essential to try to identify

' Crocco, J. A., Rooney, J. J., Fankushen, D. S., DiBenedetto, R. J., and Lyons, H. A., Archives of Internal Medicine, 1968, 121, 495 . Ehrenhaft, J. L., and Taber, R. E., Fournal of Thoracic and Cardiovascular Surgery, 1955, 30, 275

Ford, J. M., Clauss, R. H., Al Askari, S., and Wylie, R. H., fournal of Thoracic and Cardiovascular Surgery, 1958, 35, 341.

4 Linberg, E. J., American Surgeon, 1964, 30, 158

Rubin, E. H., and Rubin, M., Thoracic Diseases, 1961, p. 925. Philadelphia, Saunders.

- Fisher, A. M., and Shelley, W. M., Archives of Internal Medicine, 1966, 117, 412.

' Storey, C. F., Fournal of Thoracic and Cardiovascular Surgery, 1955, 30, 284.

- Ray, E. S., Virginia Medical Monthly, 1947, 74, 121.

- Pursel, S. E., and Lindskog, G. E., American Review of Respiratory Diseases, $1961,84,329$.

10 Randolph, H., Diseases of the Chest, 1955, 28, 416. the site and cause of bleeding, and continuous monitoring is invaluable. Adequate supplies of blood for transfusion must be made immediately available. Several forms of treatment to control bleeding have been advocated-oestrogens, vasopressin, protamine sulphate, vitamin $\mathrm{K}$, and calcium gluconate among others-but proof of their efficacy is lacking. The importance of bronchoscopy in both diagnosis and treatment is often forgotten, and there is a common misconception that bronchoscopy is valueless while the patient is bleeding. Crocco and his colleagues identified the site of bleeding at bronchoscopy in 65 out of 67 patients, and S. E. Pursel and G. E. Lindskog found the site of haemorrhage in 18 out of 21 patients who were examined while they were still bleeding. ${ }^{9}$ Bronchoscopy is also valuable in treatment, because death is frequently due to asphyxia. ${ }^{3}$ Removal of clot from bronchi is clearly important. Tracheostomy and ventilation may sometimes be required.

There is clearly a role for surgical treatment, and this is especially so if the rate of bleeding exceeds $600 \mathrm{ml}$. in 16 hours ; a previous history of significant haemoptysis may be an added indication. Haemorrhage from non-malignant lung abscess seems to be particularly lethal, and early resection of such lesions seems to improve the prognosis. ${ }^{14}$ Resection may also be safely carried out in the presence of active tuberculosis provided the patient receives effective chemotherapy cover. Massive haemoptysis occurs in some patients who are unsuitable for resection because of inadequate pulmonary reserve or other causes ; bedside determination of ventilatory function may be helpful, though it may be difficult to evaluate the results in patients with blood in the bronchial tree.

Massive haemoptysis fortunately is rare even in the experience of specialized thoracic units. It alarms patients greatly, and thus reassurance is an important part of management. But alone it is not enough, and immediate evaluation of the problem is essential, bronchoscopy occupying a central place in management. ${ }^{129}$ Timely surgical treatment, when practicable, may save many lives.

\section{Hypertension in Patients on Regular Dialysis}

Richard Bright ${ }^{1}$ first observed hypertrophied left ventricles in patients dying with terminal renal disease 133 years ago, but we have little information why hypertension appears in this state. The technique of maintenance dialysis, which prolongs life beyond terminal renal failure, has now provided information which bears on our understanding of hypertension in general.

The first and perhaps still the most startling observation was that even the most severe forms of accelerated hypertension became reversible without drugs in patients on regular dialysis. $^{2}$ This result is achieved when the intake of salt and water is rigidly restricted in adequately dialysed patients. In this situation there is a good correlation between exchangeable sodium and blood pressure. ${ }^{3}$ Within a year or two dialysis units were regarding young patients with rapid terminal deterioration in association with accelerated hypertension as the ones who would most quickly and successfully be returned to health, including a normal blood pressure, simply by adequate regular dialysis. 